\title{
Diseñadores industriales mapeando la ciudad: construyendo un diagrama físico volumétrico como estrategia colaborativa y formativa
}

Marcela B. Cabello-Mora1, Juan C. Briede-Westermeyer ${ }^{2 \star}$, Isabel M. Leal-Figueroa ${ }^{3}$ y Cristhian E. Pérez-Villalobos ${ }^{4}$ (1) Dpto. de Diseño, Facultad de Humanidades y Tecnologías de la Comunicación Social, Universidad Tecnológica Metropolitana. Dieciocho 390, Santiago - Chile (correo-e: marcela.cabello@utem.cl).

(2) Dpto. de Ingeniería en Diseño, Universidad Técnica Federico Santa María, $2^{\circ}$ piso Edificio Av. Matta \#222, Casa

Central, Valparaíso - Chile (correo-e: juancarlos.briede@usm.cl).

(3) Escuela de Diseño Gráfico, Universidad del Bío-Bío. Avda. Andrés Bello 720, Casilla 447, Chillán - Chile

(correo-e: ileal@ubiobio.cl).

(4) Departamento de Educación Médica, Universidad de Concepción, Víctor Lamas 1290, Concepción - Chile

(correo-e: cperezv@udec.cl).

* Autor a quien debe dirigirse la correspondencia.

Recibido Jul. 17, 2020; Aceptado Sep. 15, 2020; Versión final Nov. 13, 2020, Publicado Abr. 2021

\begin{abstract}
Resumen
El presente artículo explora una nueva estrategia didáctica que busca integrar la etapa inicial de trabajo individual del estudiante de diseño industrial, cuyos resultados tradicionalmente se comparten en láminas expositivas. En esta propuesta dichos resultados se representan en un volumen de diseño físico a través de trabajo colaborativo, permitiéndoles sociabilizar y obtener un material de consulta. Los estudiantes se agruparon en equipos, los cuales realizaron un estudio de campo respecto de la presencia del rubro artesanal en la ciudad de Concepción (Chile), integrándose luego en una apuesta en común: un mapa diagrama físico volumétrico de Concepción. Se aplicó una encuesta de percepción, destacándose entre los resultados la comprensión por parte de los estudiantes sobre la relevancia de trabajar por un objetivo común; sin embargo, los hallazgos evidencian que el espíritu de competencia primó por sobre la colaboración. En conclusión, esto hace suponer que la estrategia colaborativa debe iniciarse con la integración de todos los estudiantes, apuntando decisiones y acciones conjuntas como producto del consenso.
\end{abstract}

Palabras clave: diseño industrial; trabajo colaborativo; estrategia de aprendizaje; mapa objetual tridimensional

\section{Industrial designers mapping a city: constructing a physical volumetric diagram as a collaborative and formative strategy}

\begin{abstract}
This article explores a new teaching strategy that seeks to merge the initial stages of industrial design students' individual work by allowing them to socialize and obtain reference material together. The results of individual work are traditionally shared in exhibition sheets. In this proposal these results are represented, and put together through collaborative work into a physical design volume. Students were grouped in teams to conduct a field study assessing craft industry presence in the city of Concepcion (Chile). Then, students integrated their findings in a shared proposal: a physical volumetric diagram of Concepción. A perception survey was applied. The results showed that student acknowledge the relevance of working together towards a common objective. However, competitiveness prevailed over collaboration. In conclusion, this suggests that collaborative strategies should start by grouping all students with the aim of reaching a consensus for joint decisions and actions.
\end{abstract}




\section{INTRODUCCIÓN}

Este trabajo se inscribe en la lógica de la metodología de aprendizaje basado en proyectos, una estrategia común e históricamente aplicada en la formación del diseñador industrial (Yang, 2010). Ya que el desarrollo de proyectos, permite a los estudiantes desarrollar habilidades de orden superior, en lugar de memorizar la información en contextos aislados y desconectados (Majo y Baquero, 2014). La metodología de aprendizaje basado en proyectos es un paradigma de aprendizaje constructivista en el que pequeños grupos de estudiantes trabajan en colaboración para resolver problemas complejos en un contexto de proyecto del mundo real (Brodie, et. al, 2008). Demostrando ser la enseñanza- aprendizaje del diseño la mejor garantía educativa disponible hoy para el desarrollo de habilidades actitudinales y procedimentales, y lograr una mayor satisfacción asociada al aprendizaje del contenido del currículo (Gómez-Pablos et al., 2017).

En la formación del Diseñador destaca la importancia de crear una cultura de colaboración para apoyar el cambio (Fullan, 1999). Por ello, es necesario integrarlos al medio y exponerlos a problemas reales, así, mediante la resolución colaborativa de una tarea, los estudiantes tienen el reto de compartir ideas, expresar sus pensamientos y participar en la discusión (Stahl et al., 2006). Asimismo, al estimular el trabajo real con el material se genera una memoria corporal y sensorial sobre ella (Briede et al., 2016), produciéndose así una síntesis significativa que permite la posterior profundización de lo aprendido.

La enseñanza y aprendizaje del proceso de diseño depende de la filosofía de cada escuela, teniendo casos donde se aborda con el enfoque de la ingeniería del diseño (Ulrich y Eppingger, 2015), enfoques de Design Thinking (Henriksen et al, 2017) o aplicando la metodología proyectual (Munari, 2016). Este último enfoque, tradicionalmente se ha aplicado en la formación de diseño en Chile (Palmarola, 2008). Donde también se exhibe una herencia en el abordaje didáctico del proyecto de diseño, desde una aproximación exploratoria, que busca a través de la observación indagar en el contexto para reconocer, identificar, nombrar, etc.

Así, una vez definido el ámbito del trabajo, o temática del taller, es posible generar el primer encargo. Este encargo busca instar a los estudiantes a que tengan un primer acercamiento a dicho contexto, para que más adelante, conforme al desarrollo de la investigación, se articule una propuesta que responda las cuestiones claves (problemática/oportunidad) identificadas en el contexto (Alexander 1964). Este último es el caso de la Escuela de Diseño Industrial [EDI] de la Universidad del Bío-Bío, Concepción Chile, que aborda la enseñanza del diseño a través de la metodología proyectual (Munari, 2016) y la investigación convergente en sus talleres temáticos, planteados desde el inicio, a partir del estudio y observación (Scully, 2014; Martin y Hanington, 2012). Abarcando el contexto general de la temática por investigar desde un enfoque fenomenológico (Hartson y Pyla, 2012) y cuyo objetivo es conocer, explorar una temática necesaria para el diseño de productos.

La didáctica planteada por la EDI en torno a esta primera actividad es a través de una exploración de manera individual o en grupos pequeños, para posteriormente sociabilizar los resultados y retroalimentar en el aula con todos o la mayoría de los actores involucrados. Lo que se busca con este diálogo es sensibilizar al grupo curso, generando así tanto un primer acercamiento a la temática como la identificación de los factores que componen dicho contexto (tales como individuos, actividades, acciones, objetos, espacio, etc.). Esta sociabilización en aula se realiza al azar y después de que el estudiante presenta su investigación, los profesores y compañeros comentan y retroalimentan su exposición.

Bajo esta lógica, la secuencia tradicional de actividades: a) Presentación y objetivos; b) Inmersión: Salida individual a terreno para observar con pauta. c) En clase: se comparte mediante presentación y comentarios; d) Retroalimentación General: resumen de los hallazgos generales con casos particulares para ejemplificar buenas observaciones y análisis con más potencial según criterio de los docentes, también aparece la necesidad de precisar discurso, coherencia y cohesión del argumento, uso de croquis o esquemas que apoyan las observaciones, en definitiva, ayudar a la comprensión acabada de todo lo que se busca transmitir. Durante el desarrollo de los talleres, ha sido posible detectar que la etapa de socialización y sensibilización inicial es crucial para que la metodología de aprendizaje basado en proyectos sea exitosa, por lo que requiere dedicación y tiempo.

Las problemáticas que suelen surgir en la socialización: i) No es percibida significativamente dentro del proceso de diseño; ii) Generalmente son los profesores los que retroalimentan, no los estudiantes; iii) Las observaciones y croquis en muchos casos son análisis individuales, no reflexión grupal, estructurada y registrada, dificultando el establecer un análisis en profundidad de la temática que se está abordando. Si no se resuelve claramente los potenciales problemas antes descritos, los actores suelen sentirse inseguros 0 desmotivados, lo que afecta todo el proceso posterior, por ello se propone como actividad didáctica la construcción de un Diagrama Físico Volumétrico, actividad que pretende contribuir a que los estudiantes entiendan y se impliquen reflexivamente en la etapa inicial del proceso proyectual de un Taller de Diseño. 


\section{METODOLOGÍA}

Durante el año 2018, el Taller de Diseño Centrado en el Usuario [DCU], correspondiente al tercer semestre de la carrera de Diseño Industrial UBB, trabajó de manera conjunta con una asociación de artesanos de la zona, Manos del Bío-Bío. El objetivo de dicha alianza fue aportar en el proceso productivo o en el diseño de nuevos productos y de la cual se planteó la siguiente pregunta de investigación: ¿Por medio de la participación en la definición y construcción de un Diagrama Físico Volumétrico (actividad didáctica), se contribuye a que los estudiantes entiendan y se impliquen reflexivamente en la etapa inicial del proceso proyectual de un Taller de Diseño?

Acorde a la pregunta de investigación planteada y considerando los principios de Yang (2010), Majo y Baquero (2014), Fullan (1999), Stahl et al. (2006), Ulrich y Eppingger (2015), Mathias (2017) y Munari (2016), entre otros autores, el presente trabajo explora una propuesta de estrategia didáctica, que se fundamenta en la metodología de aprendizaje basado en proyectos y el trabajo colaborativo, buscando aunar y sintetizar el trabajo de la primera fase del diseño de productos: investigación en el contexto, generando un insumo de representación física. Para lo cual se utilizó una interfaz tangible y didáctica, a modo de mapa infográfico, para generar un mayor compromiso de los estudiantes en el proceso de diseño (Berglund y Grinmheden, 2011). En sentido similar, para superar el límite de no poder expresar lo que encuentran significativo en relación con el proyecto (Möller y Tollestrup, 2013) y porque el proceso de mapeo no es diferente al de lectura atenta, y eso a través de la recopilación y visualización de datos existente y las nuevas interpretaciones de la literatura pueden validarse, expandirse y disputarse (El Khatib y Schaeben, 2020). La representación tangible (lego, mapa) permite establecer un escenario y todas sus variables, (calles, vías, edificios). Esto permite contextualizar, apoyar la discusión y análisis e identificar relaciones o variables (nociones de distancia).

La relación comparativa de abordaje del Taller tradicional vs. Taller propuesto fue: a) Desarrollo Tradicional, Observación/Reflexión Individual; Materialización Croquis, láminas. En contraste a b) Desarrollo Propuesto: Observación/ Reflexión Grupal/Colectiva; Materialización Maqueta objetual. A partir de lo anterior, se plantea la pregunta de investigación: "La elaboración de una interfaz tangible permitirá la representación de conceptos abstractos, observados de manera individual por los estudiantes y facilitará la comprensión de la información compleja en un proceso iterativo de creación grupal". Para responder la pregunta de investigación, el taller DCU planteó realizar un diagnóstico colaborativo del contexto productivo consensuando e instaurando las buenas prácticas de diseño: criterios, contenidos, conocimiento.

Para ello, se dividió al grupo de estudiantes en equipos de cuatro integrantes, los cuales realizaron un estudio de campo respecto de la presencia del rubro artesanal en Concepción, investigando el contexto y luego integrando resultados con el resto de los equipos, para realizar un Mapa Diagrama Físico Volumétrico (MDFV) común (físico/análogo e interactivo por medio de elementos movibles como puntos en la ciudad y recorridos, pudiendo actualizar el diagrama para que sea fiel reflejo de la realidad estudiada) de Concepción, donde se categorizó topográficamente la oferta de artesanías, zonas de rubros y flujos peatonales en la ciudad. Este MDFV estuvo expuesto permanentemente en el aula, lo que posibilitó que fuera actualizado continuamente por los estudiantes, transformándose en un insumo de retroalimentación co-creativa para potenciar y generar una propuesta definitiva. La secuencia metodológica, para implementar esta nueva propuesta didáctica, implicó generar un mini-proyecto con la dinámica del aprendizaje basado en proyectos (Schön,1983), potenciando el trabajo colaborativo (Heck y Hallinger,2010). Etapas descritas en la Tabla 1.

Tabla 1: Etapas de la nueva didáctica propuesta para concretar el Diagrama Físico Volumétrico.

\begin{tabular}{|c|c|c|c|c|c|}
\hline I Etapa & II Etapa & III Etapa & IV Etapa & V Etapa & VI Etapa \\
\hline $\begin{array}{l}\text { Investigación } \\
\text { del contexto }\end{array}$ & $\begin{array}{l}\text { Investigación } \\
\text { específica }\end{array}$ & $\begin{array}{c}\text { Propuestas } \\
\text { grupales }\end{array}$ & Selección & $\begin{array}{l}\text { Integración de } \\
\text { criterios }\end{array}$ & Fabricación \\
\hline Individual & Equipos & Equipos & Equipos & Todos los equipos & $\begin{array}{l}\text { Todos los } \\
\text { equipos }\end{array}$ \\
\hline $\begin{array}{l}\text { Pauta de } \\
\text { observación: } \\
\text { Identificar la } \\
\text { situación, } \\
\text { actividad } \\
\text { considerando: } \\
\text { ¿qué?, } \\
\text { ¿quiénes? } \\
\text { ¿dónde?, } \\
\text { ¿cómo? }\end{array}$ & $\begin{array}{l}\text { Cada equipo ordenó } \\
\text { y clasificó la } \\
\text { información obtenida } \\
\text { Individualmente, } \\
\text { siendo ésta la } \\
\text { instancia inicial para } \\
\text { discutir los } \\
\text { hallazgos. }\end{array}$ & $\begin{array}{l}\text { Se definieron los } \\
\text { criterios y } \\
\text { requerimientos de } \\
\text { la propuesta. } \\
\text { A partir de ello } \\
\text { cada grupo } \\
\text { desarrolló } 3 \\
\text { propuestas. }\end{array}$ & $\begin{array}{l}\text { La selección se } \\
\text { realizó a través } \\
\text { de una votación, } \\
\text { con el objetivo de } \\
\text { valorar los } \\
\text { criterios } \\
\text { previamente } \\
\text { definidos. }\end{array}$ & $\begin{array}{l}\text { A partir de la } \\
\text { votación general se } \\
\text { analizaron las } \\
\text { propuestas elegidas } \\
\text { y se integraron los } \\
\text { aspectos } \\
\text { sobresalientes de } \\
\text { cada una. }\end{array}$ & $\begin{array}{l}\text { La } \\
\text { propuesta } \\
\text { final } \\
\text { contempla } \\
\text { todos los } \\
\text { aportes de } \\
\text { cada } \\
\text { equipo. }\end{array}$ \\
\hline
\end{tabular}


I Etapa: Investigación del contexto. Esta actividad de corte inductora se realizó individualmente, cada estudiante debió recorrer las calles de la ciudad y observar (quiénes, qué hacen, cómo lo hacen, en que entorno-contexto lo hacen) la presencia de artesanía en Concepción. Trabajo que el estudiante debió sociabilizar ante el resto del curso. Obteniéndose una base temática sobre la cual poder discutir y avanzar.

II Etapa: Investigación específica basada en la primera actividad. Focalizada en tópicos de la temática encontrados en el primer trabajo. El taller se dividió en 7 grupos de 4 a 5 estudiantes, los cuales cubrían los tópicos principales definidos: 1) Criterios de clasificación gráfica: utilización de croquis, esquemas y otros; 2) Criterios de clasificación temática: artesanía local / seudo artesanía / manualidad; 3) Criterios de clasificación de los materiales; 4) Ficha informativa: información relevante. Imagen de la artesanía; 5) Flujos peatonales; 6) Venta Formal e Informal y 7) Tipología de Productos -diseños / grado de autenticidad. Cada grupo debió utilizar láminas (trabajo de apuntes/observaciones asociadas) esenciales para realizar un proceso de análisis, propuesta y selección.

III Etapa: Propuestas grupales. Se definieron criterios y requerimientos básicos que debía contemplar el producto final. Cada grupo presentó tres propuestas de exhibición y clasificación conceptual utilizando bocetos y maquetas relacionadas con diversos modos para clasificar y exhibir los contenidos visualmente. Criterios:1) Integrar información de cada artesano. 2) Clasificación de rubro, materialidad, técnica y muestra del tipo de material. 3) Sección del mapa de Concepción con mayor presencia de venta de artesanía en la ciudad. 4) Elementos que permitan interactuar y actualizar la información del mapa. Hitos, flujos de peatones, zonas. Sobre requerimientos de diseño para construir el MDFV: 1) Fabricado con materiales y procesos productivos disponibles en el taller de prototipos. 2) Instalación en la sala de clases para ser consultado constantemente dentro del taller.

IV Etapa: Selección de criterios y requerimientos por medio del DATUM para evaluar las alternativas de manera objetiva. A partir de la sociabilización se generó una votación en cuanto a 1) Croquis, esquemas y otros; 2) Artesanía local / seudo artesanía / manualidad; 3) Materiales; 4) Imagen de la artesanía; 5) Flujos peatonales; 6) Venta Formal e Informal y 7) Tipología de Productos de las alternativas expuestas y así se logró la selección de la alternativa por desarrollar.

$V$ Etapa: Integración de criterios. Cada jefe de grupo debió gestionar la obtención de la información sobre 1) Croquis; 2) Artesanía; 3) Materiales; 4) Imagen; 5) Flujos peatonales; 6) Tipo de Venta y 7) Tipología de Productos de los otros grupos. Junto con ello discutieron y se llegó a consensuar los criterios constructivos y materiales para la fabricación del mapa. La integración de los elementos y subexhibidores en un único mapa infográfico de Concepción.

VI Etapa: Fabricación. Una vez que se definieron componentes, distribución final del mapa y sus subsistemas se pasó a la etapa de fabricación. En esta instancia cada equipo se organizó, y aplicando criterios de seriación lograron optimizar el proceso productivo. En todo momento, durante estas seis etapas, los estudiantes fueron retroalimentándose co-creativamente, generando en conjunto una apuesta en común sobre la clasificación de artesanías presentes en la ciudad de Concepción, útil para todo el taller.

Para la validación de la didáctica planteada y dar respuesta a la pregunta de investigación, se llevó a cabo un estudio exploratorio (Creswell, 2003) con el fin de documentar la percepción de la actividad utilizando una encuesta con 22 preguntas cerradas con escala likert entre 1 (muy en desacuerdo) y 5 (muy de acuerdo), separadas en tres temáticas: (I) Proceso de Análisis de Campo, (II) Trabajo Colaborativo y (III) Registro y visualización de información. Además de dos preguntas abiertas sobre ventajas y desventajas de la experiencia desarrollada.

\section{RESULTADOS}

Acorde a la experiencia realizada, es posible afirmar que fue posible elaborar un mapa diagrama físico volumétrico co-creativo, que quedó conformado por tres secciones informativas: a) Un Mapa de las calles del centro de la ciudad con los puntos donde se vende artesanía. Entre estos puestos se consideraron los puestos temporales, así como las tiendas formales; b) Una sección con la información de los puestos de artesanía, referida a la tipología de artesanía, dirección específica, entre otros; c) Muestrario de los materiales utilizados en dichas artesanías. En torno a la validación de la didáctica y respuesta a la pregunta de investigación, a través del estudio exploratorio sobre la percepción de los estudiantes sobre la actividad, se aplicó un cuestionario de auto respuesta, el cual fue respondido por 29 de los 34 alumnos inscritos en el taller (85\% de respuestas). Por otra parte, los resultados del mismo cuestionario en relación con el Análisis de Campo (Tabla 2), la mayoría de los estudiantes concuerda en que el trabajo de campo se realizó principalmente a través de la observación presencial (con un 52\% "De acuerdo" y un 21\% "Muy de acuerdo"), y en desacuerdo en que el trabajo de campo se haya realizado principalmente en línea (38\%); respuestas que dejan en evidencia el que culturalmente no todos los artesanos exponen sus productos a través de una vía digital y que construir un mapa de este tipo implica recorrer el territorio. También es posible afirmar que los estudiantes perciben de manera favorable el ítem: "Los criterios para el análisis de campo fueron claros y facilitaban la recopilación de datos" con un 59\%, lo que refleja la importancia de este punto para iniciar una buena recopilación. Sin 
embargo, no todos los estudiantes cumplen con el compromiso que se propone en términos de realizar primero un trabajo individual y después grupal, dado que un $34 \%$ de éstos manifiesta "en desacuerdo" con que los estudiantes trabajaron individualmente y luego se retroalimentó con el grupo.

Tabla 2. Ítem I Proceso de Análisis de Campo

\begin{tabular}{|l|c|c|c|c|c|}
\hline \multicolumn{1}{|c|}{ I. Proceso de Análisis de Campo } & 1 & 2 & 3 & 4 & 5 \\
\hline \multicolumn{1}{|c|}{${ }^{*}$ Frecuencia Mayoritaria } & $\begin{array}{c}\text { Muy en } \\
\text { desacuerdo }\end{array}$ & $\begin{array}{c}\text { En } \\
\text { desacuerdo }\end{array}$ & Neutro & $\begin{array}{c}\text { De } \\
\text { acuerdo }\end{array}$ & $\begin{array}{c}\text { Muy de } \\
\text { acuerdo }\end{array}$ \\
\hline $\begin{array}{l}\text { 1. Los criterios para el análisis de campo eran claros y } \\
\text { facilitaban la recopilación de datos. }\end{array}$ & $\begin{array}{c}0 \\
(0 \%)\end{array}$ & $\begin{array}{c}3 \\
(10 \%)\end{array}$ & $\begin{array}{c}8 \\
(28 \%)\end{array}$ & $\begin{array}{c}17 \\
(59 \%)^{*}\end{array}$ & $\begin{array}{c}1 \\
(3 \%)\end{array}$ \\
\hline $\begin{array}{l}\text { 2. El trabajo de campo se realizó principalmente a través } \\
\text { de la observación presencial. }\end{array}$ & $\begin{array}{c}1 \\
(3 \%)\end{array}$ & $\begin{array}{c}5 \\
(17 \%)\end{array}$ & $\begin{array}{c}2 \\
(7 \%)\end{array}$ & $\begin{array}{c}15 \\
(52 \%)^{*}\end{array}$ & $\begin{array}{c}6 \\
(21 \%)\end{array}$ \\
\hline 3. El trabajo de campo se realizó principalmente online. & 5 & 11 & 7 & 5 & 1 \\
& $(17 \%)$ & $(38 \%)^{*}$ & $(24 \%)$ & $(17 \%)$ & $(3 \%)$ \\
\hline $\begin{array}{l}\text { 4. El trabajo de campo se realizó individualmente y luego } \\
\text { se retroalimentó con el grupo. }\end{array}$ & $\begin{array}{c}3 \\
(10 \%)\end{array}$ & $\begin{array}{c}10 \\
(34 \%)^{*}\end{array}$ & $\begin{array}{c}6 \\
(21 \%)\end{array}$ & $\begin{array}{c}7 \\
(24 \%)\end{array}$ & $\begin{array}{c}3 \\
(10 \%)\end{array}$ \\
\hline $\begin{array}{l}\text { 5. El tiempo asignado permitió alcanzar una amplitud y } \\
\text { profundidad del trabajo de campo asignado. }\end{array}$ & 0 & 5 & 9 & 12 & 3 \\
$(10 \%)$ & $(17 \%)$ & $(31 \%)$ & $(41 \%)^{*}$ & $(10 \%)$ \\
\hline
\end{tabular}

Según los resultados relacionados con las preguntas descritas en la Tabla 3 sobre el Trabajo Colaborativo, es posible encontrar que la mayoría de los estudiantes se manifiesta de forma positiva en su actuar respecto del trabajo colaborativo, de forma que las opciones "de acuerdo" y "muy de acuerdo" suman siempre más del $70 \%$. Por ejemplo: "Compartí información relevante que recopilé individualmente con mis compañeros" (83\%); "Argumenté adecuadamente mis ideas ante mis compañeros" (72\%); "Consideré los aportes de mis compañeros" (93\%); "Retroalimenté a mis compañeros sobre sus ideas" (76\%); "En el grupo fueron respetadas las opiniones personales" (76\%); "En el grupo las decisiones se tomaron por consenso" (79\%), y "Respeté las opiniones que eran diferentes a la mía" (97\%). También, existe un grupo no menor que se manifiesta neutro con las siguientes afirmaciones referidas a la elección de compañeros al momento de formar grupo de trabajo: "Traté de trabajar más con las personas que me caen bien" (52\%); "Evité trabajar con las personas que me caen mal" (52\%), lo que mostraría cierto grado de madurez o bien simplemente indiferencia. Situación que se complementa con que el hecho de que el ítem "compartir y retroalimentar los hallazgos con mis compañeros permitió identificar criterios y clasificaciones que orientaron el trabajo", tuvo un total de $66 \%$ a favor, sin embargo, un $28 \%$ muestra una postura neutral, dejando una incertidumbre que se aclara con las respuestas a preguntas abiertas más adelante.

Respecto de los resultados expuestos en relación a las preguntas planteadas al "Registro y Visualización de Información" es posible afirmar que un alto porcentaje de estudiantes está "de acuerdo" en que los temas se registraron con coherencia entre sí (69\%), y que las afirmaciones que siguen esta misma tendencia de evaluación positiva son las referidas a la facilidad de registro de los temas y la profundidad alcanzada que permitió el tiempo y la disposición superando el $50 \%$ de acuerdo en ambos casos. Pese a lo anterior, hay un grupo no menor que se muestra imparcial cuando se les consulta sobre la visualización del registro en el mapa, su facilidad y coherencia, lo que indica una posible falla técnica o falta de interés en los aspectos formales del trabajo de parte de los estudiantes.

En la documentación de respuestas a preguntas abiertas, referidas a ventajas y aportes del trabajo realizado, se confirma que el ejercicio de mapeo fue recibido de manera positiva por parte de los estudiantes, como también lo relacionado al trabajo de "análisis de campo" (Tabla 2) y "registro y visualización de la información" (Tabla 4). Al contrario de lo relacionado con el "trabajo colaborativo", donde es posible apreciar que algunos aspectos del trabajo en equipo no quedan tan claros con las preguntas cerradas, donde es posible apreciar, por ejemplo, que la mayoría de los estudiantes cree que el mayor problema o desventaja del trabajo fue la existencia de rivalidad entre compañeros, debido a que no se respetó el trabajo de sus pares al momento de construir el mapa.

En relación con comentarios hechos por estudiantes sobre el cambio de formato bidimensional a tridimensional sobre sus procesos cognitivos. "La ventaja y aporte de esta actividad fue la fácil ubicación de los puestos de artesanía y no artesanía en Concepción, ya que primero caminamos por las calles para encontrar a los artesanos y los locales de materias primas y luego seleccionamos qué poner" Estudiante 1. "Participar en la maqueta del mapa de Concepción me dio una idea más clara de los diferentes puntos de comercialización. Además, esta investigación me permitió aprender de los artesanos y como se desenvuelven en la zona." Estudiante 2. "Fue fácil definir cómo mostrar la información que juntamos como equipo, ya que maqueteábamos los volúmenes al tiro", Estudiante 3. 
Tabla 3. Ítem II Trabajo Colaborativo. *Frecuencia Mayoritaria

\begin{tabular}{|c|c|c|c|c|c|}
\hline Trabajo colaborativo & 1 & 2 & 3 & 4 & 5 \\
\hline & $\begin{array}{c}\text { Muy en } \\
\text { desacuerdo }\end{array}$ & $\begin{array}{c}\text { En } \\
\text { desacuerdo }\end{array}$ & Neutro & $\begin{array}{c}\text { De } \\
\text { acuerdo }\end{array}$ & $\begin{array}{l}\text { Muy de } \\
\text { acuerdo }\end{array}$ \\
\hline $\begin{array}{l}\text { 1. El trabajo colaborativo fue coherente con lo } \\
\text { establecido en el encargo. }\end{array}$ & $\begin{array}{c}1 \\
(3 \%)\end{array}$ & $\begin{array}{c}6 \\
(21 \%)\end{array}$ & $\begin{array}{c}11 \\
(38 \%)^{*}\end{array}$ & $\begin{array}{c}10 \\
(34 \%)\end{array}$ & $\begin{array}{c}1 \\
(3 \%)\end{array}$ \\
\hline $\begin{array}{l}\text { 2. Compartí información relevante que recopilé } \\
\text { individualmente con mis compañeros. }\end{array}$ & $\begin{array}{c}0 \\
0 \\
(0 \%)\end{array}$ & $\begin{array}{c}2 \\
(7 \%)\end{array}$ & $\begin{array}{c}3 \\
(10 \%)\end{array}$ & $\begin{array}{c}16 \\
(55 \%)^{*}\end{array}$ & $\begin{array}{c}8 \\
(28 \%)\end{array}$ \\
\hline $\begin{array}{l}\text { 3. Compartir y retroalimentar los hallazgos con mis } \\
\text { compañeros permitió identificar criterios y } \\
\text { clasificaciones que orientaron el trabajo. }\end{array}$ & $\begin{array}{c}1 \\
(3 \%)\end{array}$ & $\begin{array}{c}1 \\
(3 \%)\end{array}$ & $\begin{array}{c}8 \\
(28 \%)\end{array}$ & $\begin{array}{c}11 \\
(38 \%)^{*}\end{array}$ & $\begin{array}{c}8 \\
(28 \%)\end{array}$ \\
\hline $\begin{array}{l}\text { 4. Argumenté adecuadamente mis ideas ante mis } \\
\text { compañeros. }\end{array}$ & $\begin{array}{c}0 \\
(0 \%)\end{array}$ & $\begin{array}{c}1 \\
(3 \%)\end{array}$ & $\begin{array}{c}7 \\
(24 \%)\end{array}$ & $\begin{array}{c}16 \\
(55 \%)^{*}\end{array}$ & $\begin{array}{c}5 \\
(17 \%)\end{array}$ \\
\hline 5. Consideré los aportes de mis compañeros. & $\begin{array}{c}0 \\
(0 \%)\end{array}$ & $\begin{array}{c}1 \\
(3 \%)\end{array}$ & $\begin{array}{c}1 \\
(3 \%)\end{array}$ & $\begin{array}{c}17 \\
(59 \%)^{*}\end{array}$ & $\begin{array}{c}10 \\
(34 \%)\end{array}$ \\
\hline $\begin{array}{ll}\text { 6. Retroalimenté a mis compañeros sobre sus } \\
\text { ideas. }\end{array}$ & $\begin{array}{c}0 \\
(0 \%) \\
\end{array}$ & $\begin{array}{c}1 \\
(3 \%) \\
\end{array}$ & $\begin{array}{c}6 \\
(21 \%) \\
\end{array}$ & $\begin{array}{c}18 \\
(62 \%)^{*}\end{array}$ & $\begin{array}{c}4 \\
(14 \%) \\
\end{array}$ \\
\hline $\begin{array}{l}\text { 7. En el grupo fueron respetadas las opiniones } \\
\text { personales. }\end{array}$ & $\begin{array}{c}1 \\
(3 \%) \\
\end{array}$ & $\begin{array}{c}2 \\
(7 \%) \\
\end{array}$ & $\begin{array}{c}4 \\
(14 \%) \\
\end{array}$ & $\begin{array}{c}15 \\
(52 \%)^{*}\end{array}$ & $\begin{array}{c}7 \\
(24 \%) \\
\end{array}$ \\
\hline $\begin{array}{l}\text { 8. En el grupo las decisiones se tomaron por } \\
\text { consenso. }\end{array}$ & $\begin{array}{c}2 \\
(7 \%) \\
\end{array}$ & $\begin{array}{c}3 \\
(10 \%)\end{array}$ & $\begin{array}{c}1 \\
(3 \%)\end{array}$ & $\begin{array}{c}13 \\
(45 \%)^{*}\end{array}$ & $\begin{array}{c}10 \\
(34 \%)\end{array}$ \\
\hline 9. En el grupo fue fácil compartir ideas. & $\begin{array}{c}3 \\
(10 \%) \\
\end{array}$ & $\begin{array}{c}6 \\
(21 \%) \\
\end{array}$ & $\begin{array}{c}7 \\
(24 \%) \\
\end{array}$ & $\begin{array}{c}5 \\
(17 \%) \\
\end{array}$ & $\begin{array}{c}8 \\
(28 \%)^{*}\end{array}$ \\
\hline $\begin{array}{l}\text { 10. Respeté las opiniones que eran diferentes a la } \\
\text { mía. }\end{array}$ & $\begin{array}{c}1 \\
(3 \%)\end{array}$ & $\begin{array}{c}0 \\
(0 \%)\end{array}$ & $\begin{array}{c}0 \\
(0 \%)\end{array}$ & $\begin{array}{c}17 \\
(59 \%)^{*}\end{array}$ & $\begin{array}{c}11 \\
(38 \%)\end{array}$ \\
\hline $\begin{array}{l}\text { 11. Traté de trabajar más con las personas que me } \\
\text { caen bien. }\end{array}$ & $\begin{array}{c}4 \\
(14 \%)\end{array}$ & $\begin{array}{c}2 \\
(7 \%)\end{array}$ & $\begin{array}{c}15 \\
(52 \%)^{*}\end{array}$ & $\begin{array}{c}4 \\
(14 \%)\end{array}$ & $\begin{array}{c}4 \\
(14 \%)\end{array}$ \\
\hline $\begin{array}{l}\text { 12. Evité trabajar con las personas que me caen } \\
\text { mal. }\end{array}$ & $\begin{array}{c}5 \\
(17 \%)\end{array}$ & $\begin{array}{c}4 \\
(14 \%)\end{array}$ & $\begin{array}{c}15 \\
(52 \%)^{*}\end{array}$ & $\begin{array}{c}1 \\
(3 \%)\end{array}$ & $\begin{array}{c}4 \\
(14 \%)\end{array}$ \\
\hline
\end{tabular}

Asimismo, la falta de comunicación y organización entre grupos provocó incoherencia en algunas partes del trabajo grupal, lo que reforzaría las respuestas neutras relacionadas con las conformaciones de equipo y contrasta notoriamente con la alta autoevaluación que cada uno se adjudica sobre estos mismos puntos. A partir de lo anterior, se podría inferir que los estudiantes trabajaron bien dentro de cada grupo porque trabajaron con compañeros afines, pero no superaron la competitividad que se produce al trabajar entre grupos para lograr el objetivo final.

Tabla 4. Ítem III Registro y Visualización de Información

\begin{tabular}{|c|c|c|c|c|c|}
\hline $\begin{array}{l}\text { Registro y visualización de } \\
\text { información }\end{array}$ & 1 & 2 & 3 & 4 & 5 \\
\hline${ }^{*}$ Frecuencia Mayoritaria & $\begin{array}{c}\text { Muy en } \\
\text { desacuerdo }\end{array}$ & $\begin{array}{c}\text { En } \\
\text { desacuerdo }\end{array}$ & Neutro & $\begin{array}{l}\text { De } \\
\text { acuerdo }\end{array}$ & $\begin{array}{l}\text { Muy de } \\
\text { acuerdo }\end{array}$ \\
\hline $\begin{array}{l}\text { 13. Los temas a analizar se registraron con } \\
\text { facilidad. }\end{array}$ & $\begin{array}{c}0 \\
(0 \%)\end{array}$ & $\begin{array}{c}4 \\
(14 \%)\end{array}$ & $\begin{array}{c}9 \\
(31 \%)\end{array}$ & $\begin{array}{c}14 \\
(48 \%)^{*}\end{array}$ & $\begin{array}{c}2 \\
(7 \%)\end{array}$ \\
\hline $\begin{array}{l}\text { 14. Los temas se registraron con coherencia } \\
\text { entre sí. }\end{array}$ & $\begin{array}{c}0 \\
(0 \%)\end{array}$ & $\begin{array}{c}2 \\
(7 \%)\end{array}$ & $\begin{array}{c}7 \\
(24 \%)\end{array}$ & $\begin{array}{c}19 \\
(66 \%)^{*}\end{array}$ & $\begin{array}{c}1 \\
(3 \%)\end{array}$ \\
\hline $\begin{array}{l}\text { 15. Los temas se analizaron con profundidad que } \\
\text { permitió el tiempo y la disposición. }\end{array}$ & $\begin{array}{c}0 \\
(0 \%)\end{array}$ & $\begin{array}{c}4 \\
(14 \%)\end{array}$ & $\begin{array}{c}7 \\
(24 \%)\end{array}$ & $\begin{array}{c}15 \\
(52 \%)^{*}\end{array}$ & $\begin{array}{c}3 \\
(10 \%)\end{array}$ \\
\hline $\begin{array}{l}\text { 16. Fue fácil definir cómo visualizar/mostrar la } \\
\text { información en el Mapa/Objeto. }\end{array}$ & $\begin{array}{c}2 \\
(7 \%)\end{array}$ & $\begin{array}{c}7 \\
(24 \%)\end{array}$ & $\begin{array}{c}13 \\
(45 \%)^{*}\end{array}$ & $\begin{array}{c}6 \\
(21 \%)\end{array}$ & $\begin{array}{c}1 \\
(3 \%)\end{array}$ \\
\hline $\begin{array}{l}\text { 17. El registro fue coherente con la forma en que } \\
\text { se visualizó/mostró la información }\end{array}$ & $\begin{array}{c}0 \\
(0 \%)\end{array}$ & $\begin{array}{c}1 \\
(3 \%)\end{array}$ & $\begin{array}{c}14 \\
(48 \%)^{*}\end{array}$ & $\begin{array}{c}13 \\
(45 \%)\end{array}$ & $\begin{array}{c}1 \\
(3 \%)\end{array}$ \\
\hline
\end{tabular}

Finalmente, cuando se les pregunta sobre las ventajas de dicho trabajo, la categoría más destacada fue la fácil ubicación de los puestos de artesanía y no artesanía en Concepción, algo evidente por el objetivo del trabajo. Además, se encontraron factores que dificultaron el trabajo tales como la cantidad de integrantes, la disposición del artesano para avanzar con la investigación y tomar una decisión como curso al finalizar el trabajo (Mapa Diagrama Físico Volumétrico de Concepción). 


\section{DISCUSIÓN}

Respecto a la didáctica planteada en el taller DCU, en relación con la generación de una interfaz tangible para facilitar la comprensión de información compleja en un proceso de creación grupal, es posible identificar que la elaboración de un Mapa Diagrama Físico Volumétrico permite agilizar el proceso exploratorio de acercamiento a la temática del taller durante la etapa inicial del mismo, porque desde su elaboración en el Análisis de Campo, ítem I, surgen a lo menos tres secciones informativas: a) Planimetría y localización topológica de los actores sobre los cuales se pretende trabajar; b) Indicaciones de las variables temporales de cada hito relevante de los actores; c) Visión general de la información anteriormente descrita, que permite categorizar y entregar datos relevantes sobre las tipologías de estudio observadas; d) Relaciones territoriales entre los actores de estudio y entre los potenciales participantes dentro del territorio que no han sido consideradas pero que pueden ser involucradas.

Compartir esta información y retroalimentarse del trabajo de los compañeros de su mismo grupo permite llegar a una instancia de reflexión más amplia. Situación similar a la descrita por Hey et al. (2007), en el sentido de que cuando los miembros del equipo se toman el tiempo para discutir y seleccionar un objetivo compartido, adaptan sus criterios y crean una referencia común en la dirección del proyecto. En cuanto al Registro y Visualización de Información, ítem III, será relevante facilitar este aspecto clave, pudiendo ser mejorado a través plataformas de fácil acceso para los actores del proyecto, asimismo mejorar el panorama visual favorece la lectura global de la información. En lo referido al Ítem II, Trabajo Colaborativo, fortalecer la sinergia que se espera entre grupos es complejo, ya que se debe superar la cultura competitiva, que provoca falta de comunicación y organización entre grupos. Esto genera incoherencia en algunas partes del trabajo del taller, entre las acciones y la percepción de irregularidad o desnivelación en el trabajo de unos grupos en relación a otros. Situación reflejada en las respuestas neutras sobre las conformaciones de equipo y el notorio contraste entre éstas, asimismo con las respuestas asociadas a la autoevaluación que cada uno se adjudica sobre su participación en dichos grupos.

De lo anterior, es plausible inferir que los alumnos trabajaron bien dentro de cada grupo porque trabajaron con compañeros afines, pero no superaron la competitividad que se produce al trabajar entre grupos para lograr el objetivo final. En relación a lo descrito en párrafos anteriores y lo planteado por autores como Stahl et al., (2006), Gómez-Pablos et al., (2017) y Baker et al, (2017), sobre la participación de los estudiantes en discusiones de proyectos en una cultura de formación colaborativa en la enseñanza del diseño, es posible afirmar que esto efectivamente permite generar sinergias creativas que van en pro del desarrollo de actividades actitudinales y procedimentales respecto del contenido del taller, pero que la efectividad de éstas dependerá del nivel de madurez de los estudiantes, tanto de la capacidad de aportar a otros como de autoevaluar su desempeño. En este caso se observó que los grupos de estudiantes se conformaron con base en afinidad y que la rivalidad entre grupos complicó la toma de decisiones al momento de configurar el Mapa Diagrama Físico Volumétrico, ya que dicha rivalidad generaba que entre grupos no se respetara el trabajo de pares, primando intereses relacionados a la competencia.

\section{CONCLUSIONES}

La metodología de aprendizaje basado en proyectos es, sin duda, dentro de la enseñanza del diseño, la didáctica más usada y efectiva, porque favorece el aprendizaje significativo. Realizar un MDFV co-creativo ayuda a este proceso, porque permite visualizar el registro de la información y su análisis, introduce el trabajo por realizar facilitando su comprensión, como también, motivando y mejorando la comunicación entre los actores. Es una estrategia efectiva, pero debe efectuarse de manera continua, es decir el aporte para el estudiante estará dado por la realización de actividades similares en todo el proceso formativo. Además, la didáctica empleada permitió constatar que la actividad colaborativa tiene potencial para fortalecer las competencias relacionadas con la capacidad de análisis, síntesis, autogestión, indagación y presentación que se esperan de un estudiante de diseño cursando el tercer semestre de carrera, especialmente en los puntos del actuar investigativo y reflexivo. Igualmente, antes de aplicar este tipo de estrategias colaborativas se requiere que los estudiantes puedan aprender a dejar de lado sus intereses personales en post de un objetivo común consensuado y discutido. Como muchos otros aprendizajes, debe ser ejercitado a lo largo de todo el proceso formativo, especialmente en los primeros años para sentar las bases metodológicas aplicables en años posteriores. Sobre el gran porcentaje de estudiantes con dudas de cómo abordar esta actividad, es posible concluir que la efectividad de estas estrategias dependerá también de la capacidad de comunicar y sintetizar la información, aspectos que deberían ser incluidos en la didáctica del tema que aborda cada taller, ya que son aspectos fundamentales no solo para el ejercicio del oficio, sino, además, para el desarrollo de proyectos colaborativos e interdisciplinarios, que son los escenarios laborales a los cuales todo diseñador se verá enfrentado. En cuanto al trabajo en terreno, sigue siendo relevante cuando se vincula con la construcción del modelo físico mapa porque da las perspectivas, entornos y volúmenes experimentados por los estudiantes, un modelo físico entonces, se presenta como una conexión entre la vivencia fenomenológica y la posterior reflexión conceptual, participación del cuerpo que abre el espacio al aprendizaje significativo. 


\section{REFERENCIAS}

Alexander, C., Notes on the Synthesis of Form. Harvard Paperbacks. Harvard University Press; Underlining edition, 224 páginas. (1964)

Baker, A., Lin, T., Chen, J., Paul, N., Anderson R. y Nguyen-Jahiel, K., Effects of Teacher Framing on Student Engagement during Collaborative Reasoning Discussions. Doi 10.1016/j.cedpsych.2017.08.007.Contemporary Educational Psychology. 51. (2017).

Berglund, A. y Grimheden, M., The Importance of Prototyping for Education in Product Innovation Engineering. In ICORD 11: Proceedings of the 3rd International Conference on Research into Design Engineering, Bangalore, India 10 al 12 de enero (2011).

Briede, J.C., Leal, I.M. y Van der Linden, J., The matter of illusion; Revista 180 ISSN 0718-2309 (37), 32-35. (2016)

Brodie, L., Hong, Z., Anthony, G., Steps in Developing an Advanced Software Engineering Course using Problem Based Learning. Doi 10.11120/ened.2008.03010002 Engineering Education, 3 (2008), pp. 2 12, (2008)

Creswell, J.W., Research Design: Qualitative, Quantitative and Mixed Methods Approaches, 4a ed. Sage Publications S.A. USA (2003).

El Khatib, R. y Schaeben, M. , Why Map Literature? Geospatial Prototyping for Literary Studies and Digital Humanities. Doi 10.16995/dscn.330 Digital Studies/Le champ numérique. 10. (2020)

Frascara, J., Design Education, Training, and the Broad Picture: Eight Experts Respond to a Few Questions, doi.10.1016/j.sheji.2019.12.003. She Ji: The Journal of Design, Economics, and Innovation, 6(1), 106-117, (2020)

Fullan, M.G. , Change forces: The sequel. Falmer, Londres, (1999).

Gómez-Pablos, V.B., Martín del Pozo, M. y García-Valcárcel A. , Project-Based Learning (PBL) Through the Incorporation of Digital Technologies: An Evaluation Based on the Experience of Serving Teachers. Doi 10.1016/j.chb.2016.11.056. Computers in Human Behavior, 68, 501-512 (2017).

Hartson R. y Pyla P.S., Design Thinking, Ideation and Sketching; The UX Book: Process and Guidelines for Ensuring a Quality User Experience, 1a ed., pp 251-297, Morgan Kaufmann, USA (2012).

Heck, R.H. y Hallinger, P. , Collaborative Leadership Effects on School Improvement: Integrating Unidirectional- and ReciprocalEffects Models. Doi 10.1086/656299 The Elementary School Journal111(2), 226-252 (2010).

Henriksen, D., Richardson, C., y Mehta, R., Design thinking: A Creative Approach to Educational Problems of Practice. Doi.10.1016/j.tsc.2017.10.001 26, 140-153. Thinking Skills and Creativity. (2017).

Hey, J., Joyce, C. y Beckman, S., Framing Innovation: Negotiating Shared Frames During Early Design Phases. Doi 10.1504/JDR.2007.015564. Journal of Design Research. 6. (2007).

Majo, F. y Baqueró, M. ,8 ideas clave: Los proyectos interdisciplinarios, $1^{\text {a }}$ edición, Grao, Barcelona, (2014).

Martin, B. y Hanington, B.M. , Universal Methods of Design: 100 Ways to Research Complex Problems, Develop Innovative Ideas, and Design Effective Solutions, 120-121. Rockport Publisher, Beverly, USA. (2012).

Mathias, D.,Evolving LEGO: Prototyping Requirements for a Customizable Construction kit. In: Proceedings of the 21st International Conference on Engineering Design (ICED17), Vol. 4: Design Methods and Tools, Vancouver, Canada, 21 -al 25 de agosto (2017).

Møller, L., Tollestrup, C. ,Creating Shared Understanding in Product Development Teams. How to 'Build the Beginning'. Doi 10.1007/978-1-4471-4180-8 ISBN : 978-1-4471-4179-2 Springer-Verlag London. 134 Pages. (2013)

Munari, B. „¿Cómo nacen los objetos? .Editorial Gustavo Gili. 296 páginas. Barcelona (2016)

Noël, G. ,We All Want High-Quality Design Education: But What Might That Mean?. Doi 10.1016/j.sheji.2020.02.003. She Ji: The Journal of Design, Economics, and Innovation. 6. 5-12. (2020).

Palmarola, H. ,Capítulo: Chile. Diseño industrial en historia del diseño en América Latina y el Caribe, Industrialización y comunicación visual para la autonomía. Coordinadores: Silvia Fernández, Gui Bonsiepe, Editora Edgard Blücher, San Pablo, Brasil, (2008).

Schön, D.A. ,The Reflective Practitioner: How Professionals Think in Action, Harper Collins, New York, Estados Unidos(1983).

Scully, A., Observation: Listen with Our Eyes and Look with Our Ears. Proceedings16th International conference on Engineering and Product Design Education (E\&PDE14), Design Education and Human Technology Relations, 592-598, University of Twente, The Netherlands, United Kingdom,4-5 de September (2014).

Stahl, G., Koschmann, T. y Suthers, D. ,Computer-Supported Collaborative Learning. In R. Sawyer (Ed.), The Cambridge Handbook of the Learning Sciences, 409-426, University Cambridge Press, Cambridge, England (2006).

Ulrich, K. y Eppinger, S. ,Product Design and Development, 6a ed. McGraw-Hill Higher Education, DF, México. (2015)

Yang, N.B. ,Applying Project-Based Learning to Product Design, International Conference on System Science, Engineering Design and Manufacturing Informatization, vol.1, 171-173, DC, USA, 12-14 de November (2010). 